\title{
A regularization projection algorithm for various problems with nonlinear mappings in Hilbert spaces
}

\author{
Buthinah A Bin Dehaish', Abdul Latif², Huda O Bakodah' and Xiaolong Qin²*
}

"Correspondence: qxIxajh@163.com ${ }^{2}$ Department of Mathematics, King Abdulaziz University, P.O. Box 80203, Jeddah, 21589, Saudi Arabia Full list of author information is available at the end of the article

\begin{abstract}
In this paper, a regularization projection algorithm is investigated for solving common elements of an equilibrium problem, a variational inequality problem and a fixed point problem of a strictly pseudocontractive mapping. Strong convergence theorems are established in the framework of real Hilbert spaces.
\end{abstract}

Keywords: Hilbert space; equilibrium problem; variational inequality; nonexpansive mapping; fixed point

\section{Introduction and preliminaries}

Let $H$ be a real Hilbert space with the inner product $\langle\cdot, \cdot\rangle$ and the norm $\|\cdot\|$. Let $C$ be a nonempty closed convex subset of $H . P_{C}$ stands for the metric projection from $H$ onto $C$. Let $F$ be a bifunction of $C \times C$ into $\mathbb{R}$, where $\mathbb{R}$ denotes the set of real numbers. Consider the following equilibrium problem in the terminology of Blum and Oettli [1]:

$$
\text { Find } x \in C \text { such that } F(x, y) \geq 0, \quad \forall y \in C \text {. }
$$

In this paper, the solution set of problem (1.1) is denoted by $\operatorname{FP}(F)$.

To study problem (1.1), we may assume that $F$ satisfies the following conditions:

(A1) $F(x, x)=0$ for all $x \in C$;

(A2) $F$ is monotone, i.e., $F(x, y)+F(y, x) \leq 0$ for all $x, y \in C$;

(A3) for each $x, y, z \in C$,

$$
\limsup _{t \downarrow 0} F(t z+(1-t) x, y) \leq F(x, y)
$$

(A4) for each $x \in C, y \mapsto F(x, y)$ is convex and lower semi-continuous.

Let $S$ be a self-mapping of $C . F(S)$ stands for the fixed point set of $S$. Recall that $S$ is said to be $\beta$-contractive if there exists a constant $\beta \in[0,1)$ such that

$$
\|S x-S y\| \leq \beta\|x-y\|, \quad \forall x, y \in C .
$$

$S$ is said to be nonexpansive if

$$
\|S x-S y\| \leq\|x-y\|, \quad \forall x, y \in C .
$$

o 2015 Bin Dehaish et al.; licensee Springer. This is an Open Access article distributed under the terms of the Creative Commons Attribution License (http://creativecommons.org/licenses/by/4.0), which permits unrestricted use, distribution, and reproduction in any medium, provided the original work is properly credited. 
$S$ is said to be $\kappa$-strictly pseudocontractive if there exists a constant $\kappa \in[0,1)$ such that

$$
\|S x-S y\|^{2} \leq\|x-y\|^{2}+\kappa\|(x-S x)-(y-S y)\|^{2}, \quad \forall x, y \in C .
$$

The class of $\kappa$-strictly pseudocontractive mappings was introduced by Browder and Petryshyn [2]. It is clear that every nonexpansive mapping is a 0 -strictly pseudocontractive mapping.

Let $A: C \rightarrow H$ be a mapping. Recall that $A$ is said to be monotone if

$$
\langle A x-A y, x-y\rangle \geq 0, \quad \forall x, y \in C .
$$

$A$ is said to be strongly monotone if there exists a constant $\alpha>0$ such that

$$
\langle A x-A y, x-y\rangle \geq \alpha\|x-y\|^{2}, \quad \forall x, y \in C .
$$

For such a case, we say that $A$ is $\alpha$-strongly monotone. $A$ is said to be inverse-strongly monotone if there exists a constant $\alpha>0$ such that

$$
\langle A x-A y, x-y\rangle \geq \alpha\|A x-A y\|^{2}, \quad \forall x, y \in C .
$$

For such a case, we say that $A$ is $\alpha$-inverse-strongly monotone. It is clear that $A$ is $\alpha$-inverse-strongly monotone if and only if $A^{-1}$ is $\alpha$-strongly monotone.

Recall that the classical variational inequality is to find $x \in C$ such that

$$
\langle A x, y-x\rangle \geq 0, \quad \forall y \in C
$$

It is known that $x \in C$ is a solution of (1.2) if and only if $x$ is a fixed point of $P_{C}(I-r A)$, where $r>0$ is a constant and $I$ is an identity mapping. From now on, the solution set of (1.2) is denoted by $\mathrm{VI}(C, A)$.

A set-valued mapping $T: H \rightarrow 2^{H}$ is said to be monotone if for all $x, y \in H, f \in T x$ and $g \in$ Ty imply $\langle x-y, f-g\rangle \geq 0$. A monotone mapping $T: H \rightarrow 2^{H}$ is maximal if the graph $G(T)$ of $T$ is not properly contained in the graph of any other monotone mapping. It is known that a monotone mapping $T$ is maximal if and only if, for any $(x, f) \in H \times H,\langle x-y, f-g\rangle \geq 0$ for all $(y, g) \in G(T)$ implies $f \in T x$.

Recently, Iiduka and Takahshi [3] investigated variational inequality (1.2) and fixed points of a nonexpansive mapping based on a Halpern-like algorithm. To be more clear, they proved the following result.

Theorem IT Let $C$ be a closed convex subset of a real Hilbert space $H$. Let $A$ be an $\alpha$-inverse-strongly monotone mapping of $C$ into $H$, and let $S$ be a nonexpansive mapping of $C$ into itself such that $F(S) \cap \operatorname{VI}(C, A) \neq \emptyset$. Suppose $x_{1}=x \in C$ and $\left\{x_{n}\right\}$ is given by

$$
x_{n+1}=\alpha_{n} x+\left(1-\alpha_{n}\right) S P_{C}\left(x_{n}-\lambda_{n} A x_{n}\right)
$$

for every $n=1,2, \ldots$, where $\left\{\alpha_{n}\right\}$ is a sequence in $[0,1)$ and $\left\{\lambda_{n}\right\}$ is a sequence in $[0,2 \alpha]$. If $\left\{\alpha_{n}\right\}$ and $\left\{\lambda_{n}\right\}$ are chosen so that $\lambda_{n} \in[a, b]$ for some $a, b$ with $0<a<b<2 \alpha, \lim _{n \rightarrow \infty} \alpha_{n}=0$, $\sum_{n=1}^{\infty} \alpha_{n}=\infty, \sum_{n=1}^{\infty}\left|\alpha_{n+1}-\alpha_{n}\right|<\infty$ and $\sum_{n=1}^{\infty}\left|\lambda_{n+1}-\lambda_{n}\right|<\infty$, then $\left\{x_{n}\right\}$ converges strongly to $P_{F(S) \cap \mathrm{VI}(C, A)} x$. 
Since equilibrium problem (1.1) provides a unified model of several problems such as variational inequalities, fixed point problems and inclusion problems. In [4], Takahashi and Takahashi further studied fixed points of a nonexpansive mapping and equilibrium problem (1.1) based on the viscosity approximation method, which was introduced by Moudafi [5]. To be more clear, they proved the following result.

Theorem TT Let $C$ be a nonempty closed convex subset of a real Hilbert space H. Let $F$ be a bifunction from $C \times C$ to $R$ satisfying (A1)-(A4), and let $S$ be a nonexpansive mapping of $C$ into $H$ such that $F(S) \cap \mathrm{EP}(F) \neq \emptyset$. Let $f$ be a contraction of $H$ into itself, and let $\left\{x_{n}\right\}$ and $\left\{u_{n}\right\}$ be sequences generated by $x_{1} \in H$ and

$$
\left\{\begin{array}{l}
F\left(u_{n}, y\right)+\frac{1}{r_{n}}\left\langle y-u_{n}, u_{n}-x_{n}\right\rangle \geq 0, \quad \forall y \in C, \\
x_{n+1}=\alpha_{n} f\left(x_{n}\right)+\left(1-\alpha_{n}\right) S u_{n}
\end{array}\right.
$$

for every $n=1,2, \ldots$, where $\left\{\alpha_{n}\right\} \subset[0,1]$ and $\left\{r_{n}\right\} \subset(0, \infty)$ satisfy $\lim _{n \rightarrow \infty} \alpha_{n}=0, \sum_{n=1}^{\infty} \alpha_{n}=$ $\infty, \sum_{n=1}^{\infty}\left|\alpha_{n+1}-\alpha_{n}\right|<\infty, \liminf _{n \rightarrow \infty} r_{n}>0$ and $\sum_{n=1}^{\infty}\left|r_{n+1}-r_{n}\right|<\infty$. Then $\left\{x_{n}\right\}$ and $\left\{u_{n}\right\}$ converge strongly to $z \in F(S) \cap \operatorname{EP}(F)$, where $z=P_{F(S) \cap \mathrm{EP}(F)} f(z)$.

Subsequently, many authors investigated common solution problems based on hybrid projection methods due to real world applications, for example, image restoration and digital signal processing; see [6-20] and the references therein. In view of the complexity of convex, hybrid projection methods, they are not easy to implement. In this paper, we study a regularization projection algorithm for solving equilibrium problem (1.1), variational inequality (1.2) and a fixed point problem of a $\kappa$-strictly pseudocontractive mapping. Possible computation errors are taken into account. Strong convergence theorems are established in the framework of real Hilbert spaces.

In order to prove our main results, we also need the following lemmas.

Lemma 1.1 [21] Let $C$ be a nonempty closed convex subset of $H$. Let $A$ be a monotone mapping of $C$ into $H$, and let $N_{C} v$ be a normal cone to $C$ at $v \in C$, i.e.,

$$
N_{C} v=\{w \in H:\langle v-u, w\rangle \geq 0, \forall u \in C\}
$$

and define a mapping $T$ on $C$ by

$$
T v= \begin{cases}A v+N_{C} v, & v \in C, \\ \emptyset, & v \notin C .\end{cases}
$$

Then $T$ is maximal monotone and $0 \in T v$ if and only if $\langle A v, u-v\rangle \geq 0$ for all $u \in C$.

Lemma 1.2 [2] Let $C$ be a nonempty closed convex subset of $H$, and let $S: C \rightarrow H$ be a $\kappa$-strictly pseudocontractive mapping. Then $I-S$ is demi-closed at zero.

Lemma 1.3 [1] Let $C$ be a nonempty closed convex subset of $H$, and let $F: C \times C \rightarrow \mathbb{R}$ be a bifunction satisfying (A1)-(A4). Then, for any $r>0$ and $x \in H$, there exists $z \in C$ such that

$$
F(z, y)+\frac{1}{r}\langle y-z, z-x\rangle \geq 0, \quad \forall y \in C .
$$


Further, define

$$
T_{r} x=\left\{z \in C: F(z, y)+\frac{1}{r}\langle y-z, z-x\rangle \geq 0, \forall y \in C\right\}
$$

for all $r>0$ and $x \in H$. Then the following hold:

(a) $T_{r}$ is single-valued;

(b) $T_{r}$ is firmly nonexpansive, i.e., for any $x, y \in H$,

$$
\left\|T_{r} x-T_{r} y\right\|^{2} \leq\left\langle T_{r} x-T_{r} y, x-y\right\rangle
$$

(c) $F\left(T_{r}\right)=\mathrm{EP}(F)$;

(d) $\mathrm{EP}(F)$ is closed and convex.

Lemma 1.4 [2] Let $C$ be a nonempty closed convex subset of $H$, and let $S: C \rightarrow H$ be a $\kappa$-strictly pseudocontractive mapping. Define a mapping $T$ by $T=\delta I+(1-\delta) S$, where $\delta$ is a constant in $(0,1)$. If $\delta \in[\kappa, 1)$ then $T$ is nonexpansive and $F(T)=F(S)$.

Lemma 1.5 [22] Let $\left\{x_{n}\right\}$ and $\left\{y_{n}\right\}$ be bounded sequences in $H$, and let $\left\{\beta_{n}\right\}$ be a sequence in $[0,1]$ with $0<\liminf _{n \rightarrow \infty} \beta_{n} \leq \limsup _{n \rightarrow \infty} \beta_{n}<1$. Suppose $x_{n+1}=\left(1-\beta_{n}\right) y_{n}+\beta_{n} x_{n}$ for all integers $n \geq 0$ and

$$
\limsup _{n \rightarrow \infty}\left(\left\|y_{n+1}-y_{n}\right\|-\left\|x_{n+1}-x_{n}\right\|\right) \leq 0 .
$$

Then $\lim _{n \rightarrow \infty}\left\|y_{n}-x_{n}\right\|=0$.

Lemma 1.6 [23] Assume that $\left\{\alpha_{n}\right\}$ is a sequence of nonnegative real numbers such that

$$
\alpha_{n+1} \leq\left(1-\gamma_{n}\right) \alpha_{n}+\delta_{n}+\mu_{n}
$$

where $\left\{\gamma_{n}\right\}$ is a sequence in $(0,1)$ and $\left\{\mu_{n}\right\},\left\{\delta_{n}\right\}$ are real sequences such that

(i) $\sum_{n=1}^{\infty} \gamma_{n}=\infty$ and $\sum_{n=1}^{\infty} \mu_{n}<\infty$;

(ii) $\lim \sup _{n \rightarrow \infty} \delta_{n} / \gamma_{n} \leq 0$ or $\sum_{n=1}^{\infty}\left|\delta_{n}\right|<\infty$.

Then $\lim _{n \rightarrow \infty} \alpha_{n}=0$.

\section{Main results}

Theorem 2.1 Let $C$ be a closed convex subset of a real Hilbert space H. Let $A: C \rightarrow H$ be an $\alpha$-inverse-strongly monotone mapping, and let $F$ be a bifunction from $C \times C$ to $\mathbb{R}$ which satisfies (A1)-(A4). Let $S: C \rightarrow H$ be a $\kappa$-strictly pseudocontractive mapping, and let $f$ be a $\beta$-contraction on $H$. Assume that $\Omega=F(S) \cap \operatorname{VI}(C, A) \cap \operatorname{EP}(F) \neq \emptyset$. Let $\left\{r_{n}\right\}$ and $\left\{s_{n}\right\}$ be positive real number sequences. Let $\left\{\alpha_{n}\right\},\left\{\beta_{n}\right\},\left\{\gamma_{n}\right\}$ and $\left\{\delta_{n}\right\}$ be real number sequences in $(0,1)$ such that $\alpha_{n}+\beta_{n}+\gamma_{n}=1$. Let $\left\{x_{n}\right\}$ be a sequence generated in the following process:

$$
\left\{\begin{array}{l}
x_{1} \in H \\
F\left(z_{n}, z\right)+\frac{1}{r_{n}}\left\langle z-z_{n}, z_{n}-x_{n}\right\rangle \geq 0, \quad \forall z \in C, \\
y_{n}=P_{C}\left(z_{n}-s_{n} A z_{n}+e_{n}\right) \\
x_{n+1}=\alpha_{n} f\left(x_{n}\right)+\beta_{n} x_{n}+\gamma_{n}\left(\delta_{n} y_{n}+\left(1-\delta_{n}\right) S y_{n}\right),
\end{array}\right.
$$


where $\left\{e_{n}\right\}$ is a sequence in $H$. Assume that the control sequences satisfy the following conditions:

(a) $\lim _{n \rightarrow \infty} \alpha_{n}=0$ and $\sum_{n=1}^{\infty} \alpha_{n}=\infty$;

(b) $0<\liminf _{n \rightarrow \infty} \beta_{n} \leq \limsup _{n \rightarrow \infty} \beta_{n}<1$;

(c) $\sum_{n=1}^{\infty}\left\|e_{n}\right\|<\infty, \lim _{n \rightarrow \infty}\left|\delta_{n+1}-\delta_{n}\right|=0$ and $\kappa \leq \delta_{n} \leq \delta<1$;

(d) $\lim _{n \rightarrow \infty}\left|r_{n+1}-r_{n}\right|=0$ and $\liminf _{n \rightarrow \infty} r_{n}>0$;

(e) $\lim _{n \rightarrow \infty}\left|s_{n+1}-s_{n}\right|=0,0<s \leq s_{n} \leq s^{\prime}<2 \alpha$,

where $\delta, s, s^{\prime}$ are real constants. Then $\left\{x_{n}\right\}$ converges strongly to $q$, which is also a unique solution to the variational inequality

$$
\langle f(x)-x, x-y\rangle \geq 0, \quad \forall y \in C .
$$

Proof For any $x, y \in C$, we see that

$$
\begin{aligned}
& \left\|\left(I-s_{n} A\right) x-\left(I-s_{n} A\right) y\right\|^{2} \\
& \quad=\|x-y\|^{2}-2 s_{n}\langle x-y, A x-A y\rangle+s_{n}^{2}\|A x-A y\|^{2} \\
& \quad \leq\|x-y\|^{2}-s_{n}\left(2 \alpha-s_{n}\right)\|A x-A y\|^{2} .
\end{aligned}
$$

By using condition (e), we see that $\left\|\left(I-s_{n} A\right) x-\left(I-s_{n} A\right) y\right\| \leq\|x-y\|$. This proves that $I-s_{n} A$ is nonexpansive. Put $S_{n}=\delta_{n} I+\left(1-\delta_{n}\right) S$. It follows from Lemma 1.4 that $S_{n}$ is nonexpansive and $F\left(S_{n}\right)=F(S)$. Let $p \in \Omega$ be fixed arbitrarily. Hence, we have

$$
\begin{aligned}
\left\|x_{n+1}-p\right\| & \leq \alpha_{n}\left\|f\left(x_{n}\right)-p\right\|+\beta_{n}\left\|x_{n}-p\right\|+\gamma_{n}\left\|S_{n} y_{n}-p\right\| \\
& \leq \alpha_{n} \beta\left\|x_{n}-p\right\|+\alpha_{n}\|f(p)-p\|+\beta_{n}\left\|x_{n}-p\right\|+\gamma_{n}\left\|y_{n}-p\right\| \\
& \leq\left(1-\alpha_{n}(1-\beta)\right)\left\|x_{n}-p\right\|+\alpha_{n}\|f(p)-p\|+\left\|e_{n}\right\| \\
& \leq \max \left\{\left\|x_{n}-p\right\|, \frac{\|f(p)-p\|}{1-\beta}\right\}+\left\|e_{n}\right\| .
\end{aligned}
$$

It follows that $\left\|x_{n}-p\right\| \leq \max \left\{\left\|x_{1}-p\right\|, \frac{\|f(p)-p\|}{1-\beta}\right\}+\sum_{n=1}^{\infty}\left\|e_{n}\right\|$. This shows that $\left\{x_{n}\right\}$ is bounded, so are $\left\{y_{n}\right\}$ and $\left\{z_{n}\right\}$. Let $\lambda_{n}=\frac{x_{n+1}-\beta_{n} x_{n}}{1-\beta_{n}}$. It follows that

$$
\begin{aligned}
\lambda_{n+1}-\lambda_{n}= & \frac{\alpha_{n+1} f\left(x_{n+1}\right)+\gamma_{n+1} S_{n+1} y_{n+1}}{1-\beta_{n+1}}-\frac{\alpha_{n} f\left(x_{n}\right)+\gamma_{n} S_{n} y_{n}}{1-\beta_{n}} \\
= & \frac{\alpha_{n+1}\left(f\left(x_{n+1}\right)-S_{n+1} y_{n+1}\right)+\left(1-\beta_{n+1}\right) S_{n+1} y_{n}}{1-\beta_{n+1}} \\
& -\frac{\alpha_{n}\left(f\left(x_{n}\right)-S_{n} y_{n}\right)+\left(1-\beta_{n}\right) S_{n} y_{n}}{1-\beta_{n}} \\
= & \frac{\alpha_{n+1}\left(f\left(x_{n+1}\right)-S_{n+1} y_{n+1}\right)}{1-\beta_{n+1}}-\frac{\alpha_{n}\left(f\left(x_{n}\right)-S_{n} y_{n}\right)}{1-\beta_{n}}+S_{n+1} y_{n+1}-S_{n} y_{n} .
\end{aligned}
$$

Hence, we have

$$
\begin{aligned}
\left\|\lambda_{n+1}-\lambda_{n}\right\| \leq & \frac{\alpha_{n+1}\left\|f\left(x_{n+1}\right)-S_{n+1} y_{n+1}\right\|}{1-\beta_{n+1}}+\frac{\alpha_{n}\left\|f\left(x_{n}\right)-S_{n} y_{n}\right\|}{1-\beta_{n}} \\
& +\left\|S_{n+1} y_{n+1}-S_{n} y_{n}\right\| .
\end{aligned}
$$


Since $z_{n}=T_{r_{n}} x_{n}$, we find that $F\left(z_{n}, z\right)+\frac{1}{r_{n}}\left\langle z-z_{n}, z_{n}-x_{n}\right\rangle \geq 0, \forall z \in C$ and $F\left(z_{n+1}, z\right)+$ $\frac{1}{r_{n+1}}\left\langle z-z_{n+1}, z_{n+1}-x_{n+1}\right\rangle \geq 0, \forall z \in C$. It follows that $F\left(z_{n}, z_{n+1}\right)+\frac{1}{r_{n}}\left\langle z_{n+1}-z_{n}, z_{n}-x_{n}\right\rangle \geq 0$ and $F\left(z_{n+1}, z_{n}\right)+\frac{1}{r_{n+1}}\left\langle z_{n}-z_{n+1}, z_{n+1}-x_{n+1}\right\rangle \geq 0, \forall z \in C$. By using condition (A2), we find that $\left\langle z_{n+1}-z_{n}, \frac{z_{n}-x_{n}}{r_{n}}-\frac{z_{n+1}-x_{n+1}}{r_{n+1}}\right\rangle \geq 0$. Hence, we have

$$
\left\langle z_{n+1}-z_{n}, z_{n+1}-x_{n}-\frac{r_{n}}{r_{n+1}}\left(z_{n+1}-x_{n+1}\right)\right\rangle \geq\left\|z_{n+1}-z_{n}\right\|^{2} .
$$

This implies that $\left\langle z_{n+1}-z_{n}, x_{n+1}-x_{n}+\left(1-\frac{r_{n}}{r_{n+1}}\right)\left(z_{n+1}-x_{n+1}\right)\right\rangle \geq\left\|z_{n+1}-z_{n}\right\|^{2}$. Hence, we have

$$
\left\|z_{n+1}-z_{n}\right\| \leq\left\|x_{n+1}-x_{n}\right\|+\frac{\left|r_{n+1}-r_{n}\right|}{r_{n+1}}\left\|T_{r_{n+1}} x_{n}-x_{n+1}\right\| .
$$

It follows that

$$
\begin{aligned}
\left\|y_{n+1}-y_{n}\right\| \leq & \left\|P_{C}\left(z_{n+1}-s_{n+1} A z_{n+1}+e_{n+1}\right)-P_{C}\left(z_{n}-s_{n+1} A z_{n}+e_{n+1}\right)\right\| \\
& +\left\|P_{C}\left(z_{n}-s_{n+1} A z_{n}+e_{n+1}\right)-P_{C}\left(z_{n}-s_{n} A z_{n}+e_{n}\right)\right\| \\
\leq & \left\|\left(I-s_{n+1} A\right) z_{n+1}-\left(I-s_{n+1} A\right) z_{n}\right\| \\
& \quad+\left\|\left(z_{n}-s_{n+1} A z_{n}+e_{n+1}\right)-\left(z_{n}-s_{n} A z_{n}+e_{n}\right)\right\| \\
\leq & \left\|z_{n+1}-z_{n}\right\|+\left|s_{n+1}-s_{n}\right|\left\|A z_{n}\right\|+\left\|e_{n+1}\right\|+\left\|e_{n}\right\| \\
\leq & \left\|x_{n+1}-x_{n}\right\|+\frac{\left|r_{n+1}-r_{n}\right|}{r_{n+1}}\left\|T_{r_{n+1}} x_{n}-x_{n}\right\| \\
& +\left|s_{n+1}-s_{n}\right|\left\|A z_{n}\right\|+\left\|e_{n+1}\right\|+\left\|e_{n}\right\| .
\end{aligned}
$$

This implies that

$$
\begin{aligned}
& \left\|S_{n+1} y_{n+1}-S_{n} y_{n}\right\| \\
& \leq\left\|S_{n+1} y_{n+1}-S_{n+1} y_{n}\right\|+\left\|S_{n+1} y_{n}-S_{n} y_{n}\right\| \\
& \leq\left\|y_{n+1}-y_{n}\right\|+\left\|S_{n+1} y_{n}-S_{n} y_{n}\right\| \\
& \leq\left\|x_{n+1}-x_{n}\right\|+\frac{\left|r_{n+1}-r_{n}\right|}{r_{n+1}}\left\|T_{r_{n+1}} x_{n}-x_{n}\right\| \\
& +\left|s_{n+1}-s_{n}\right|\left\|A z_{n}\right\|+\left\|e_{n+1}\right\|+\left\|e_{n}\right\|+\left|\delta_{n+1}-\delta_{n}\right||| S y_{n}-y_{n} \| .
\end{aligned}
$$

Substituting (2.2) into (2.1), we find that

$$
\begin{aligned}
& \left\|\lambda_{n+1}-\lambda_{n}\right\|-\left\|x_{n+1}-x_{n}\right\| \\
& \leq \frac{\alpha_{n+1}\left\|f\left(x_{n+1}\right)-S_{n+1} y_{n+1}\right\|}{1-\beta_{n+1}}+\frac{\alpha_{n}\left\|f\left(x_{n}\right)-S_{n} y_{n}\right\|}{1-\beta_{n}} \\
& \quad+\frac{\left|r_{n+1}-r_{n}\right|}{r_{n+1}}\left\|T_{r_{n+1}} x_{n}-x_{n}\right\| \\
& \quad+\left|s_{n+1}-s_{n}\right|\left\|A z_{n}\right\|+\left\|e_{n+1}\right\|+\left\|e_{n}\right\|+\left|\delta_{n+1}-\delta_{n}\right|\left\|S y_{n}-y_{n}\right\| .
\end{aligned}
$$

It follows from conditions (a)-(e) that

$$
\limsup _{n \rightarrow \infty}\left(\left\|\lambda_{n+1}-\lambda_{n}\right\|-\left\|x_{n+1}-x_{n}\right\|\right) \leq 0 .
$$


By using Lemma 1.5 , we see that $\lim _{n \rightarrow \infty}\left\|\lambda_{n}-x_{n}\right\|=0$, which in turn implies that

$$
\lim _{n \rightarrow \infty}\left\|x_{n+1}-x_{n}\right\|=0
$$

Since $T_{r_{n}}$ is firmly nonexpansive, we find that

$$
\begin{aligned}
\left\|z_{n}-p\right\|^{2} & =\left\|T_{r_{n}} x_{n}-T_{r_{n}} p\right\|^{2} \\
& \leq\left\langle x_{n}-p, z_{n}-p\right\rangle \\
& =\frac{1}{2}\left(\left\|x_{n}-p\right\|^{2}+\left\|z_{n}-p\right\|^{2}-\left\|x_{n}-z_{n}\right\|^{2}\right) .
\end{aligned}
$$

That is,

$$
\left\|z_{n}-p\right\|^{2} \leq\left\|x_{n}-p\right\|^{2}-\left\|x_{n}-z_{n}\right\|^{2}
$$

It follows that

$$
\begin{aligned}
\left\|x_{n+1}-p\right\|^{2} & \leq \alpha_{n}\left\|f\left(x_{n}\right)-p\right\|^{2}+\beta_{n}\left\|x_{n}-p\right\|^{2}+\gamma_{n}\left\|S_{n} y_{n}-p\right\|^{2} \\
& \leq \alpha_{n}\left\|f\left(x_{n}\right)-p\right\|^{2}+\beta_{n}\left\|x_{n}-p\right\|^{2}+\gamma_{n}\left\|y_{n}-p\right\|^{2} \\
& \leq \alpha_{n}\left\|f\left(x_{n}\right)-p\right\|^{2}+\beta_{n}\left\|x_{n}-p\right\|^{2}+\gamma_{n}\left\|z_{n}-p\right\|^{2}+f_{n} \\
& \leq \alpha_{n}\left\|f\left(x_{n}\right)-p\right\|^{2}+\left\|x_{n}-p\right\|^{2}-\gamma_{n}\left\|x_{n}-z_{n}\right\|^{2}+f_{n},
\end{aligned}
$$

where $f_{n}=\left\|e_{n}\right\|\left(\left\|e_{n}\right\|+2\left\|z_{n}-p\right\|\right)$. This further implies that

$$
\begin{aligned}
\gamma_{n}\left\|x_{n}-z_{n}\right\|^{2} & \leq \alpha_{n}\left\|f\left(x_{n}\right)-p\right\|^{2}+\left\|x_{n}-p\right\|^{2}-\left\|x_{n+1}-p\right\|^{2}+f_{n} \\
& \leq \alpha_{n}\left\|f\left(x_{n}\right)-p\right\|^{2}+\left(\left\|x_{n}-p\right\|+\left\|x_{n+1}-p\right\|\right)\left\|x_{n}-x_{n+1}\right\|+f_{n} .
\end{aligned}
$$

By using conditions (a) and (b), we find from (2.3) that

$$
\lim _{n \rightarrow \infty}\left\|z_{n}-x_{n}\right\|=0
$$

Since $A$ is $\alpha$-inverse-strongly monotone, we find that

$$
\begin{aligned}
\left\|y_{n}-p\right\|^{2} & \leq\left\|\left(z_{n}-s_{n} A z_{n}\right)-\left(p-s_{n} A p\right)+e_{n}\right\|^{2} \\
& \leq\left\|\left(z_{n}-p\right)-s_{n}\left(A z_{n}-A p\right)\right\|^{2}+f_{n} \\
& \leq\left\|x_{n}-p\right\|^{2}-s_{n}\left(2 \alpha_{n}-s_{n}\right)\left\|A z_{n}-A p\right\|^{2}+f_{n} .
\end{aligned}
$$

It follows that

$$
\begin{aligned}
\left\|x_{n+1}-p\right\|^{2} & \leq \alpha_{n}\left\|f\left(x_{n}\right)-p\right\|^{2}+\beta_{n}\left\|x_{n}-p\right\|^{2}+\gamma_{n}\left\|S_{n} y_{n}-p\right\|^{2} \\
& \leq \alpha_{n}\left\|f\left(x_{n}\right)-p\right\|^{2}+\beta_{n}\left\|x_{n}-p\right\|^{2}+\gamma_{n}\left\|y_{n}-p\right\|^{2} \\
& \leq \alpha_{n}\left\|f\left(x_{n}\right)-p\right\|^{2}+\left\|x_{n}-p\right\|^{2}-s_{n}\left(2 \alpha-s_{n}\right) \gamma_{n}\left\|A z_{n}-A p\right\|^{2}+f_{n} .
\end{aligned}
$$


This yields that

$$
s_{n}\left(2 \alpha-s_{n}\right) \gamma_{n}\left\|A z_{n}-A p\right\|^{2} \leq \alpha_{n}\left\|f\left(x_{n}\right)-p\right\|^{2}+\left\|x_{n}-p\right\|^{2}-\left\|x_{n+1}-p\right\|^{2}+f_{n} \text {. }
$$

By using (2.3), we find from conditions (a), (c) and (d) that

$$
\lim _{n \rightarrow \infty}\left\|A z_{n}-A p\right\|=0
$$

Since $P_{C}$ is firmly nonexpansive, we find that

$$
\begin{aligned}
\left\|y_{n}-p\right\|^{2} \leq & \left\langle\left(I-s_{n} A\right) z_{n}+e_{n}-\left(I-s_{n} A\right) p, y_{n}-p\right\rangle \\
= & \frac{1}{2}\left\{\left\|\left(I-s_{n} A\right) z_{n}+e_{n}-\left(I-s_{n} A\right) p\right\|^{2}+\left\|y_{n}-p\right\|^{2}\right. \\
& \left.-\left\|\left(I-s_{n} A\right) z_{n}+e_{n}-\left(I-s_{n} A\right) p-\left(y_{n}-p\right)\right\|^{2}\right\} \\
\leq & \frac{1}{2}\left\{\left\|z_{n}-p\right\|^{2}+f_{n}+\left\|y_{n}-p\right\|^{2}-\left\|z_{n}-y_{n}-\left(s_{n}\left(A z_{n}-A p\right)-e_{n}\right)\right\|^{2}\right\} \\
= & \frac{1}{2}\left\{\left\|z_{n}-p\right\|^{2}+f_{n}+\left\|y_{n}-p\right\|^{2}-\left\|z_{n}-y_{n}\right\|^{2}\right. \\
& \left.+2\left\langle z_{n}-y_{n}, s_{n}\left(A z_{n}-A p\right)-e_{n}\right\rangle-\left\|s_{n}\left(A z_{n}-A p\right)-e_{n}\right\|^{2}\right\} \\
\leq & \frac{1}{2}\left\{\left\|x_{n}-p\right\|^{2}+f_{n}+\left\|y_{n}-p\right\|^{2}-\left\|z_{n}-y_{n}\right\|^{2}\right. \\
& \left.+2\left\|z_{n}-y_{n}\right\|\left\|s_{n}\left(A z_{n}-A p\right)-e_{n}\right\|-\left\|s_{n}\left(A z_{n}-A p\right)-e_{n}\right\|^{2}\right\},
\end{aligned}
$$

which yields that

$$
\begin{aligned}
\left\|y_{n}-p\right\|^{2} \leq & \left\|x_{n}-p\right\|^{2}+f_{n}-\left\|z_{n}-y_{n}\right\|^{2}+2 s_{n}\left\|z_{n}-y_{n}\right\|\left\|A z_{n}-A p\right\| \\
& +2\left\|z_{n}-y_{n}\right\|\left\|e_{n}\right\| .
\end{aligned}
$$

It follows that

$$
\begin{aligned}
\left\|x_{n+1}-p\right\|^{2} \leq & \alpha_{n}\left\|f\left(x_{n}\right)-p\right\|^{2}+\beta_{n}\left\|x_{n}-p\right\|^{2}+\gamma_{n}\left\|S_{n} y_{n}-p\right\|^{2} \\
\leq & \alpha_{n}\left\|f\left(x_{n}\right)-p\right\|^{2}+\beta_{n}\left\|x_{n}-p\right\|^{2}+\gamma_{n}\left\|y_{n}-p\right\|^{2} \\
\leq & \alpha_{n}\left\|f\left(x_{n}\right)-p\right\|^{2}+\left\|x_{n}-p\right\|^{2}+f_{n}-\gamma_{n}\left\|z_{n}-y_{n}\right\|^{2} \\
& +2 s_{n} \gamma_{n}\left\|z_{n}-y_{n}\right\|\left\|A z_{n}-A p\right\|+2\left\|z_{n}-y_{n}\right\|\left\|e_{n}\right\| .
\end{aligned}
$$

This in turn leads to

$$
\begin{aligned}
\gamma_{n}\left\|z_{n}-y_{n}\right\|^{2} \leq & \alpha_{n}\left\|f\left(x_{n}\right)-p\right\|^{2}+\left\|x_{n}-p\right\|^{2}-\left\|x_{n+1}-p\right\|^{2}+f_{n} \\
& +2 s_{n} \gamma_{n}\left\|z_{n}-y_{n}\right\|\left\|A z_{n}-A p\right\|+2\left\|z_{n}-y_{n}\right\|\left\|e_{n}\right\| .
\end{aligned}
$$

By use of (2.3) and (2.5), we find from restrictions (a), (b), (c) and (e) that

$$
\lim _{n \rightarrow \infty}\left\|z_{n}-y_{n}\right\|=0
$$


On the other hand, we have

$$
\gamma_{n}\left\|S_{n} y_{n}-x_{n}\right\| \leq\left\|x_{n+1}-x_{n}\right\|+\alpha_{n}\left\|x_{n}-f\left(x_{n}\right)\right\|
$$

By using conditions (a) and (b), we find from (2.3) that

$$
\lim _{n \rightarrow \infty}\left\|x_{n}-S_{n} y_{n}\right\|=0
$$

Since $S_{n}$ is nonexpansive, we find that

$$
\begin{aligned}
\left\|S_{n} x_{n}-x_{n}\right\| & \leq\left\|S_{n} x_{n}-S_{n} y_{n}\right\|+\left\|S_{n} y_{n}-x_{n}\right\| \\
& \leq\left\|x_{n}-z_{n}\right\|+\left\|z_{n}-y_{n}\right\|+\left\|S_{n} y_{n}-x_{n}\right\| .
\end{aligned}
$$

It follows from (2.4), (2.6) and (2.7) that

$$
\lim _{n \rightarrow \infty}\left\|x_{n}-S_{n} x_{n}\right\|=0
$$

Notice that

$$
\begin{aligned}
\left\|S x_{n}-x_{n}\right\| & \leq\left\|S x_{n}-\left(\delta_{n} x_{n}+\left(1-\delta_{n}\right) S x_{n}\right)\right\|+\left\|S_{n} x_{n}-x_{n}\right\| \\
& \leq \delta_{n}\left\|S x_{n}-x_{n}\right\|+\left\|S_{n} x_{n}-x_{n}\right\| .
\end{aligned}
$$

By using condition (c), we find that

$$
\lim _{n \rightarrow \infty}\left\|x_{n}-S x_{n}\right\|=0
$$

Now, we are in a position to show $\lim \sup _{n \rightarrow \infty}\left\langle f(q)-q, x_{n}-q\right\rangle \leq 0$, where $q=P_{\Omega} f(q)$. To show it, we can choose a subsequence $\left\{x_{n_{i}}\right\}$ of $\left\{x_{n}\right\}$ such that

$$
\limsup _{n \rightarrow \infty}\left\langle f(q)-q, x_{n}-q\right\rangle=\lim _{i \rightarrow \infty}\left\langle f(q)-q, x_{n_{i}}-q\right\rangle
$$

Since $\left\{x_{n_{i}}\right\}$ is bounded, we can choose a subsequence $\left\{x_{n_{i j}}\right\}$ of $\left\{x_{n_{i}}\right\}$ which converges weakly to some point $\bar{x}$. We may assume, without loss of generality, that $\left\{x_{n_{i}}\right\}$ converges weakly to $\bar{x}$.

Next, we prove $\bar{x} \in \Omega$. First, we show $x \in \operatorname{EP}(F)$. Notice that

$$
F\left(y_{n}, y\right)+\frac{1}{r_{n}}\left\langle y-y_{n}, y_{n}-x_{n}\right\rangle \geq 0, \quad \forall y \in C .
$$

By using condition (A2), we see that $\frac{1}{r_{n}}\left\langle y-y_{n}, y_{n}-x_{n}\right\rangle \geq F\left(y, y_{n}\right), \forall y \in C$. Replacing $n$ by $n_{i}$, we arrive at

$$
\left\langle y-y_{n_{i}}, \frac{y_{n_{i}}-x_{n_{i}}}{r_{n_{i}}}\right\rangle \geq F\left(y, y_{n_{i}}\right), \quad \forall y \in C .
$$

By using (2.4) and (2.6), we find that $\left\{y_{n_{i}}\right\}$ converges weakly to $\bar{x}$. It follows that $0 \geq F(y, \bar{x})$. For each $t$ with $0<t \leq 1$, let $z_{t}=t z+(1-t) \bar{x}$, where $z \in C$. It follows that $z_{t} \in C$. Hence, 
we have $F\left(z_{t}, \bar{x}\right) \leq 0$. It follows that

$$
0=F\left(z_{t}, z_{t}\right) \leq t F\left(z_{t}, z\right)+(1-t) F\left(z_{t}, \bar{x}\right) \leq t F\left(z_{t}, z\right)
$$

which yields that $F\left(z_{t}, z\right) \geq 0, \forall z \in C$. Letting $t \downarrow 0$, we obtain from condition (A3) that $F(\bar{x}, z) \geq 0, \forall z \in C$. This implies that $\bar{x} \in \operatorname{EP}(F)$.

Next, we show that $\bar{x} \in \operatorname{VI}(C, A)$. Let $T$ be a maximal monotone mapping defined by

$$
T x= \begin{cases}A x+N_{C} x, & x \in C, \\ \emptyset, & x \notin C .\end{cases}
$$

For any given $(x, y) \in \operatorname{Graph}(T)$, we have $y-A x \in N_{C} x$. Since $y_{n} \in C$, we have $\left\langle x-y_{n}, y-\right.$ $A x\rangle \geq 0$. Since $y_{n}=P_{C}\left(z_{n}-s_{n} A z_{n}+e_{n}\right)$, we see that $\left\langle x-y_{n}, y_{n}-\left(I-s_{n} A\right) z_{n}-e_{n}\right\rangle \geq 0$ and hence

$$
\left\langle x-y_{n}, \frac{y_{n}-z_{n}-e_{n}}{s_{n}}+A z_{n}\right\rangle \geq 0
$$

It follows that

$$
\begin{aligned}
\left\langle x-y_{n_{i}}, y\right\rangle \geq & \left\langle x-y_{n_{i}}, A x\right\rangle \\
\geq & \left\langle x-y_{n_{i}}, A x\right\rangle-\left\langle x-y_{n_{i}}, \frac{y_{n_{i}}-z_{n_{i}}-e_{n_{i}}}{s_{n_{i}}}+A z_{n_{i}}\right\rangle \\
= & \left\langle x-y_{n_{i}}, A x-A y_{n_{i}}\right\rangle+\left\langle x-y_{n_{i}}, A y_{n_{i}}-A z_{n_{i}}\right\rangle \\
& -\left\langle x-y_{n_{i}}, \frac{y_{n_{i}}-z_{n_{i}}-e_{n_{i}}}{s_{n_{i}}}\right\rangle \\
\geq & \left\langle x-y_{n_{i}}, A y_{n_{i}}-A z_{n_{i}}\right\rangle-\left\langle x-y_{n_{i}}, \frac{y_{n_{i}}-z_{n_{i}}-e_{n_{i}}}{s_{n_{i}}}\right\rangle .
\end{aligned}
$$

Since $A$ is Lipschitz continuous, we see that $\langle x-\bar{x}, y\rangle \geq 0$. Notice that $T$ is maximal monotone and hence $0 \in T \bar{x}$. This shows that $\bar{x} \in \mathrm{VI}(C, A)$. By using Lemma 1.2, we find that $\bar{x} \in F(S)$. It follows that $\limsup _{n \rightarrow \infty}\left\langle f(q)-q, x_{n}-q\right\rangle \leq 0$,

$$
\begin{aligned}
& \left\|x_{n+1}-q\right\|^{2} \\
& \leq \alpha_{n}\left\langle f\left(x_{n}\right)-q, x_{n+1}-q\right\rangle+\beta_{n}\left\|x_{n}-q\right\|\left\|x_{n+1}-q\right\|+\gamma_{n}\left\|S_{n} y_{n}-q\right\|\left\|x_{n+1}-q\right\| \\
& \leq \alpha_{n}\left\langle f\left(x_{n}\right)-f(q), x_{n+1}-q\right\rangle+\alpha_{n}\left\langle f(q)-q, x_{n+1}-q\right\rangle+\beta_{n}\left\|x_{n}-\bar{x}\right\|\left\|x_{n+1}-q\right\| \\
& \quad+\gamma_{n}\left(\left\|x_{n}-q\right\|+e_{n}\right)\left\|x_{n+1}-q\right\| \\
& \leq \\
& \leq \frac{\alpha_{n} \beta+\beta_{n}+\gamma_{n}}{2}\left(\left\|x_{n}-q\right\|^{2}+\left\|x_{n+1}-q\right\|^{2}\right)+\alpha_{n}\left\langle f(q)-q, x_{n+1}-q\right\rangle+e_{n}\left\|x_{n+1}-q\right\| .
\end{aligned}
$$

It follows that

$$
\left\|x_{n+1}-q\right\|^{2} \leq\left(1-\alpha_{n}(1-\beta)\right)\left\|x_{n}-q\right\|^{2}+2 \alpha_{n}\left\langle f(q)-q, x_{n+1}-q\right\rangle+2 K_{n} e_{n},
$$

where $K=\sup _{n \geq 1}\left\{\left\|x_{n}-q\right\|\right\}$. By using Lemma 1.6, we find that $\lim _{n \rightarrow \infty}\left\|x_{n}-q\right\|=0$. This completes the proof. 
For nonexpansive mappings, we have the following result.

Corollary 2.2 Let $C$ be a closed convex subset of a real Hilbert space H. Let $A: C \rightarrow H$ be an $\alpha$-inverse-strongly monotone mapping, and let $F$ be a bifunction from $C \times C$ to $\mathbb{R}$ which satisfies (A1)-(A4). Let $S: C \rightarrow H$ be nonexpansive, and let $f$ be a $\beta$-contraction on $H$. Assume that $\Omega=F(S) \cap \mathrm{VI}(C, A) \cap \mathrm{EP}(F) \neq \emptyset$. Let $\left\{r_{n}\right\}$ and $\left\{s_{n}\right\}$ be positive real number sequences. Let $\left\{\alpha_{n}\right\},\left\{\beta_{n}\right\}$ and $\left\{\gamma_{n}\right\}$ be real number sequences in $(0,1)$ such that $\alpha_{n}+\beta_{n}+\gamma_{n}=1$. Let $\left\{x_{n}\right\}$ be a sequence generated in the following process:

$$
\left\{\begin{array}{l}
x_{1} \in H, \\
F\left(z_{n}, z\right)+\frac{1}{r_{n}}\left\langle z-z_{n}, z_{n}-x_{n}\right\rangle \geq 0, \quad \forall z \in C, \\
y_{n}=P_{C}\left(z_{n}-s_{n} A z_{n}+e_{n}\right) \\
x_{n+1}=\alpha_{n} f\left(x_{n}\right)+\beta_{n} x_{n}+\gamma_{n} S y_{n},
\end{array}\right.
$$

where $\left\{e_{n}\right\}$ is a sequence in $H$. Assume that the control sequences satisfy the following conditions:

(a) $\lim _{n \rightarrow \infty} \alpha_{n}=0$ and $\sum_{n=1}^{\infty} \alpha_{n}=\infty$;

(b) $0<\liminf _{n \rightarrow \infty} \beta_{n} \leq \lim \sup _{n \rightarrow \infty} \beta_{n}<1$;

(c) $\sum_{n=1}^{\infty}\left\|e_{n}\right\|<\infty$;

(d) $\lim _{n \rightarrow \infty}\left|r_{n+1}-r_{n}\right|=0$ and $\liminf _{n \rightarrow \infty} r_{n}>0$;

(e) $\lim _{n \rightarrow \infty}\left|s_{n+1}-s_{n}\right|=0,0<s \leq s_{n} \leq s^{\prime}<2 \alpha$,

where $s, s^{\prime}$ are real constants. Then $\left\{x_{n}\right\}$ converges strongly to $q$, which is also a unique solution to the variational inequality

$$
\langle f(x)-x, x-y\rangle \geq 0, \quad \forall y \in C
$$

Further, if $S$ is an identity, we have the following result.

Corollary 2.3 Let $C$ be a closed convex subset of a real Hilbert space $H$. Let $A: C \rightarrow H$ be an $\alpha$-inverse-strongly monotone mapping, and let $F$ be a bifunction from $C \times C$ to $\mathbb{R}$ which satisfies (A1)-(A4). Let $f$ be a $\beta$-contraction on $H$. Assume that $\Omega=\mathrm{VI}(C, A) \cap \mathrm{EP}(F) \neq \emptyset$. Let $\left\{r_{n}\right\}$ and $\left\{s_{n}\right\}$ be positive real number sequences. Let $\left\{\alpha_{n}\right\},\left\{\beta_{n}\right\}$ and $\left\{\gamma_{n}\right\}$ be real number sequences in $(0,1)$ such that $\alpha_{n}+\beta_{n}+\gamma_{n}=1$. Let $\left\{x_{n}\right\}$ be a sequence generated in the following process:

$$
\left\{\begin{array}{l}
x_{1} \in H, \\
F\left(z_{n}, z\right)+\frac{1}{r_{n}}\left\langle z-z_{n}, z_{n}-x_{n}\right\rangle \geq 0, \quad \forall z \in C, \\
x_{n+1}=\alpha_{n} f\left(x_{n}\right)+\beta_{n} x_{n}+\gamma_{n} P_{C}\left(z_{n}-s_{n} A z_{n}+e_{n}\right),
\end{array}\right.
$$

where $\left\{e_{n}\right\}$ is a sequence in $H$. Assume that the control sequences satisfy the following conditions:

(a) $\lim _{n \rightarrow \infty} \alpha_{n}=0$ and $\sum_{n=1}^{\infty} \alpha_{n}=\infty$;

(b) $0<\liminf _{n \rightarrow \infty} \beta_{n} \leq \lim \sup _{n \rightarrow \infty} \beta_{n}<1$;

(c) $\sum_{n=1}^{\infty}\left\|e_{n}\right\|<\infty$;

(d) $\lim _{n \rightarrow \infty}\left|r_{n+1}-r_{n}\right|=0$ and $\liminf _{n \rightarrow \infty} r_{n}>0$;

(e) $\lim _{n \rightarrow \infty}\left|s_{n+1}-s_{n}\right|=0,0<s \leq s_{n} \leq s^{\prime}<2 \alpha$, 
where $s, s^{\prime}$ are real constants. Then $\left\{x_{n}\right\}$ converges strongly to $q$, which is also a unique solution to the variational inequality

$$
\langle f(x)-x, x-y\rangle \geq 0, \quad \forall y \in C .
$$

Putting $F(x, y)=0$ and $r_{n}=1$, we find the following result.

Corollary 2.4 Let $C$ be a closed convex subset of a real Hilbert space H. Let $A: C \rightarrow H$ be an $\alpha$-inverse-strongly monotone mapping. Let $S: C \rightarrow H$ be a $\kappa$-strictly pseudocontractive mapping, and let $f$ be a $\beta$-contraction on $H$. Assume that $\Omega=F(S) \cap \operatorname{VI}(C, A) \neq \emptyset$. Let $\left\{s_{n}\right\}$ be a positive real number sequence. Let $\left\{\alpha_{n}\right\},\left\{\beta_{n}\right\},\left\{\gamma_{n}\right\}$ and $\left\{\delta_{n}\right\}$ be real number sequences in $(0,1)$ such that $\alpha_{n}+\beta_{n}+\gamma_{n}=1$. Let $\left\{x_{n}\right\}$ be a sequence generated in the following process:

$$
\left\{\begin{array}{l}
x_{1} \in H, \\
z_{n}=P_{C} x_{n}, \\
y_{n}=P_{C}\left(z_{n}-s_{n} A z_{n}+e_{n}\right), \\
x_{n+1}=\alpha_{n} f\left(x_{n}\right)+\beta_{n} x_{n}+\gamma_{n}\left(\delta_{n} y_{n}+\left(1-\delta_{n}\right) S y_{n}\right),
\end{array}\right.
$$

where $\left\{e_{n}\right\}$ is a sequence in $H$. Assume that the control sequences satisfy the following conditions:

(a) $\lim _{n \rightarrow \infty} \alpha_{n}=0$ and $\sum_{n=1}^{\infty} \alpha_{n}=\infty$;

(b) $0<\liminf _{n \rightarrow \infty} \beta_{n} \leq \limsup _{n \rightarrow \infty} \beta_{n}<1$;

(c) $\sum_{n=1}^{\infty}\left\|e_{n}\right\|<\infty, \lim _{n \rightarrow \infty}\left|\delta_{n+1}-\delta_{n}\right|=0$ and $\kappa \leq \delta_{n} \leq \delta<1$;

(d) $\lim _{n \rightarrow \infty}\left|s_{n+1}-s_{n}\right|=0,0<s \leq s_{n} \leq s^{\prime}<2 \alpha$,

where $\delta, s, s^{\prime}$ are real constants. Then $\left\{x_{n}\right\}$ converges strongly to $q$, which is also a unique solution to the variational inequality

$$
\langle f(x)-x, x-y\rangle \geq 0, \quad \forall y \in C
$$

Corollary 2.5 Let $C$ be a closed convex subset of a real Hilbert space H. Let $F$ be a bifunction from $C \times C$ to $\mathbb{R}$ which satisfies (A1)-(A4). Let $S: C \rightarrow H$ be a $\kappa$-strict pseudocontraction, and let $f$ be a $\beta$-contraction on $H$. Assume that $\Omega=F(S) \cap \mathrm{EP}(F) \neq \emptyset$. Let $\left\{r_{n}\right\}$ be a positive real number sequence. Let $\left\{\alpha_{n}\right\},\left\{\beta_{n}\right\},\left\{\gamma_{n}\right\}$ and $\left\{\delta_{n}\right\}$ be real number sequences in $(0,1)$ such that $\alpha_{n}+\beta_{n}+\gamma_{n}=1$. Let $\left\{x_{n}\right\}$ be a sequence generated in the following process:

$$
\left\{\begin{array}{l}
x_{1} \in H \\
F\left(z_{n}, z\right)+\frac{1}{r_{n}}\left\langle z-z_{n}, z_{n}-x_{n}\right\rangle \geq 0, \quad \forall z \in C \\
x_{n+1}=\alpha_{n} f\left(x_{n}\right)+\beta_{n} x_{n}+\gamma_{n}\left(\delta_{n} z_{n}+\left(1-\delta_{n}\right) S z_{n}\right)
\end{array}\right.
$$

where $\left\{e_{n}\right\}$ is a sequence in $H$. Assume that the control sequences satisfy the following conditions:

(a) $\lim _{n \rightarrow \infty} \alpha_{n}=0$ and $\sum_{n=1}^{\infty} \alpha_{n}=\infty$;

(b) $0<\liminf _{n \rightarrow \infty} \beta_{n} \leq \limsup _{n \rightarrow \infty} \beta_{n}<1$;

(c) $\sum_{n=1}^{\infty}\left\|e_{n}\right\|<\infty, \lim _{n \rightarrow \infty}\left|\delta_{n+1}-\delta_{n}\right|=0$ and $\kappa \leq \delta_{n} \leq \delta<1$;

(d) $\lim _{n \rightarrow \infty}\left|r_{n+1}-r_{n}\right|=0$ and $\liminf _{n \rightarrow \infty} r_{n}>0$, 
where $\delta$ is a real constant. Then $\left\{x_{n}\right\}$ converges strongly to $q$, which is also a unique solution to the variational inequality

$$
\langle f(x)-x, x-y\rangle \geq 0, \quad \forall y \in C .
$$

Remark 2.6 Comparing Theorem 2.1, Corollaries 2.2-2.5 with Theorems IT, TT and the corresponding results in [6-20], we have the following.

(a) We extend the nonlinear mapping from the class of nonexpansive mappings to the class of $\kappa$-strictly pseudocontractive mappings.

(b) Possible computation errors are taken into account.

(c) The conditions imposed on control sequences $\left\{\alpha_{n}\right\}$ and $\left\{s_{n}\right\}$ are relaxed.

(d) The common element is also a unique solution of variational inequality (1.2).

Theorem 2.7 Let $C$ be a closed convex subset of a real Hilbert space $H$. Let $A: C \rightarrow H$ be an $\alpha$-inverse-strongly monotone mapping, and let $f$ be a $\beta$-contraction on $H$. Assume that $\operatorname{VI}(C, A) \neq \emptyset$. Let $\left\{s_{n}\right\}$ be a positive real number sequence. Let $\left\{\alpha_{n}\right\},\left\{\beta_{n}\right\}$ and $\left\{\gamma_{n}\right\}$ be real number sequences in $(0,1)$ such that $\alpha_{n}+\beta_{n}+\gamma_{n}=1$. Let $\left\{x_{n}\right\}$ be a sequence generated in $x_{1} \in C, x_{n+1}=\alpha_{n} f\left(x_{n}\right)+\beta_{n} x_{n}+\gamma_{n} y_{n}$, where $y_{n}=P_{C}\left(x_{n}-s_{n} A x_{n}+e_{n}\right)$, where $\left\{e_{n}\right\}$ is a sequence in $H$. Assume that the control sequences satisfy the following conditions:

(a) $\lim _{n \rightarrow \infty} \alpha_{n}=0$ and $\sum_{n=1}^{\infty} \alpha_{n}=\infty$;

(b) $0<\liminf _{n \rightarrow \infty} \beta_{n} \leq \limsup _{n \rightarrow \infty} \beta_{n}<1$;

(c) $\sum_{n=1}^{\infty}\left\|e_{n}\right\|<\infty, \lim _{n \rightarrow \infty}\left|s_{n+1}-s_{n}\right|=0,0<s \leq s_{n} \leq s^{\prime}<2 \alpha$,

where $s$ and $s^{\prime}$ are real constants. Then $\left\{x_{n}\right\}$ converges strongly to $q=P_{\mathrm{VI}(C, A)} f(q)$.

Remark 2.8 To construct a mathematical model which is as close as possible to a real complex problem, we often have to use more than one constraint. Solving such problems, we have to obtain some solution which is simultaneously the solution of two or more subproblems. This is a common problem in diverse areas of mathematics and physical sciences. It consists of trying to find a solution satisfying certain constraints. In this paper, we investigate the problem of solving common solutions of an equilibrium problem, a variational inequality problem and a fixed point problem of a strictly pseudocontractive mapping based on a regularization projection algorithm. Possible computation errors are also taken into account. Strong convergence theorems are established without compact assumptions and additional metric projections in the framework of real Hilbert spaces.

Competing interests

The authors declare that they have no competing interests.

Authors' contributions

All authors have contributed equally to the work. All authors read and approved the final version of the manuscript.

\section{Author details}

'Department of Mathematics, Faculty of Science for Girls, King Abdulaziz University, P.O. Box 80203, Jeddah, 21589, Saudi Arabia. ${ }^{2}$ Department of Mathematics, King Abdulaziz University, P.O. Box 80203, Jeddah, 21589, Saudi Arabia.

\section{Acknowledgements}

This project was funded by the Deanship of Scientific Research (DSR), King Abdulaziz University under grant No. (58-130-35-HiCi). The authors, therefore, acknowledge technical and financial support of KAU. The authors are grateful to the editor and the anonymous reviewers for useful suggestions which improved the contents of the article. 
References

1. Blum, E, Oettli, W: From optimization and variational inequalities to equilibrium problems. Math. Stud. 63, 123-145 (1994)

2. Browder, FE, Petryshyn, WV: Construction of fixed points of nonlinear mappings in Hilbert space. J. Math. Anal. Appl. 20, 197-228 (1967)

3. liduka, H, Takahashi, W: Strong convergence theorems for nonexpansive mappings and inverse-strongly monotone mappings. Nonlinear Anal. 61, 341-350 (2005)

4. Takahashi, S, Takahashi, W: Viscosity approximation methods for equilibrium problems and fixed point problems in Hilbert spaces. J. Math. Anal. Appl. 331, 506-515 (2007)

5. Moudafi, A: Viscosity approximation methods for fixed-points problems. J. Math. Anal. Appl. 241, 46-55 (2000)

6. Qin, X, Shang, M, Zhou, H: Strong convergence of a general iterative method for variational inequality problems and fixed point problems in Hilbert spaces. Appl. Math. Comput. 200, 242-253 (2008)

7. Yao, Y, Yao, JC: On modified iterative method for nonexpansive mappings and monotone mappings. Appl. Math. Comput. 186, 1551-1558 (2007)

8. Cho, SY, Qin, X, Kang, SM: Iterative processes for common fixed points of two different families of mappings with applications. J. Glob. Optim. 57, 1429-1446 (2013)

9. Luo, H, Wang, Y: Iterative approximation for the common solutions of a infinite variational inequality system for inverse-strongly accretive mappings. J. Math. Comput. Sci. 2, 1660-1670 (2012)

10. Cho, SY, Li, W, Kang, SM: Convergence analysis of an iterative algorithm for monotone operators. J. Inequal. Appl. 2013, Article ID 199 (2013)

11. Zegeye, $\mathrm{H}$, Shahzad, N: Strong convergence theorem for a common point of solution of variational inequality and fixed point problem. Adv. Fixed Point Theory 2, 374-397 (2012)

12. Kim, JS, Kim, JK, Lim, WS: Convergence theorems for common solutions of various problems with nonlinear mapping J. Inequal. Appl. 2014, Article ID 2 (2014)

13. Wang, ZM, Lou, W: A new iterative algorithm of common solutions to quasi-variational inclusion and fixed point problems. J. Math. Comput. Sci. 3, 57-72 (2013)

14. Cho, SY, Qin, X: On the strong convergence of an iterative process for asymptotically strict pseudocontractions and equilibrium problems. Appl. Math. Comput. 235, 430-438 (2014)

15. Chang, SS, Lee, HWJ, Chan, CK: A new hybrid method for solving a generalized equilibrium problem, solving a variational inequality problem and obtaining common fixed points in Banach spaces, with applications. Nonlinear Anal. 73, 2260-2270 (2010)

16. Cho, SY, Kang, SM: Approximation of common solutions of variational inequalities via strict pseudocontractions. Acta Math. Sci. 32, 1607-1618 (2012)

17. $\mathrm{Wu}, \mathrm{C}, \mathrm{Liu}, \mathrm{A}$ : Strong convergence of a hybrid projection iterative algorithm for common solutions of operator equations and of inclusion problems. Fixed Point Theory Appl. 2012, Article ID 90 (2012)

18. Qin, X, Cho, YJ, Kang, SM: Viscosity approximation methods for generalized equilibrium problems and fixed point problems with applications. Nonlinear Anal. 72, 99-112 (2010)

19. Zhang, L, Tong, $\mathrm{H}$ : An iterative method for nonexpansive semigroups, variational inclusions and generalized equilibrium problems. Adv. Fixed Point Theory 4, 325-343 (2014)

20. Wang, ZM, Zhang, X: Shrinking projection methods for systems of mixed variational inequalities of Browder type, systems of mixed equilibrium problems and fixed point problems. J. Nonlinear Funct. Anal. 2014, Article ID 15 (2014)

21. Rockafellar, RT: On the maximality of sums of nonlinear monotone operators. Trans. Am. Math. Soc. 149, 75-88 (1970)

22. Suzuki, T: Strong convergence of Krasnoselskii and Mann's type sequences for one-parameter nonexpansive semigroups without Bochner integrals. J. Math. Anal. Appl. 305, 227-239 (2005)

23. Liu, LS: Ishikawa and Mann iterative process with errors for nonlinear strongly accretive mappings in Banach spaces. J. Math. Anal. Appl. 194, 114-125 (1995)

\section{Submit your manuscript to a SpringerOpen ${ }^{\circ}$ journal and benefit from:}

- Convenient online submission

- Rigorous peer review

- Immediate publication on acceptance

- Open access: articles freely available online

- High visibility within the field

- Retaining the copyright to your article 